\title{
Feasibility of monitoring stress using skin conduction measurements during intubation of newborns
}

\author{
Robin van der Lee ${ }^{1,3,4}$. Liesbeth JM Groot Jebbink ${ }^{1}$. Thea HM van Herpen ${ }^{2}$. \\ Esther J d'Haens ${ }^{1}$ • Josette Bierhuizen ${ }^{1}$ • Richard A van Lingen ${ }^{1}$
}

Received: 2 June 2015 /Revised: 9 August 2015 / Accepted: 14 August 2015 / Published online: 2 September 2015

(C) The Author(s) 2015. This article is published with open access at Springerlink.com

\begin{abstract}
The objective of this study was to assess the feasibility of monitoring stress responses in newborns during nasotracheal intubation after two different premedication regimens, using skin conductance measurements (SCM). Twenty-two newborns were randomised and premedicated with morphine+vecuronium or propofol. SCM (peaks/s) were collected prior to, during and after the procedure. Threshold for interpreting responses as stressful was 0.21 peaks/s. Intubation conditions and physiological parameters were registered. Intubation conditions were good in all newborns. Administration of morphine (range 1.4-10.3 min) before
\end{abstract}

Communicated by Peter de Winter

Robin van der Lee

R.vanderlee@amc.nl

Liesbeth JM Groot Jebbink

1.j.groot.jebbink@isala.nl

Thea HM van Herpen

t.h.m.herpen@isala.nl

Esther J d'Haens

e.j.haens@isala.nl

Josette Bierhuizen

j.bierhuizen@isala.nl

Richard A van Lingen

r.a.van.lingen@isala.nl

1 Princess Amalia Department of Paediatrics, Department of Neonatology, Isala Clinics, Dokter van Heesweg 2, 8025 AB Zwolle, The Netherlands

2 Hospital Pharmacy, Isala Clinics, Zwolle, The Netherlands

3 Department of Neonatology, Emma Children's Hospital Academic Medical Center, Amsterdam, The Netherlands

4 Present address: Department of Neonatology, Emma Children's Hospital Academic Medical Center, Meibergdreef 15,

1105AZ Amsterdam, The Netherlands administration of vecuronium did not affect SCM when a stressful stimulus was applied. Within 1.6 min (range 0.8$3 \mathrm{~min}$ ) after administration of vecuronium, SCM disappeared in 10 of 11 newborns. Propofol reduced SCM in 10 of 11 newborns at the first attempt. Further attempts were associated with increasing SCM, mostly above a threshold of 0.21 peaks/ $\mathrm{s}$. There were no significant changes in physiological parameters during the procedure for either premedication regimen.

Conclusion: The variation in SCM between individual newborns limits the usefulness of SCM as stress monitor during intubation. The use of neuromuscular blockers for premedication precludes monitoring of SCM completely in newborns.

What is Known:

- Skin conductance measurements have been used successfully to monitor pain in awake newborn infants.

What is New:

- Premedicated newborns display significant interindividual variation in skin conductance measurements during an intubation procedure.

- Neuromuscular blockade causes skin conductance measurements to disappear completely.

Keywords Galvanic skin response · Deep sedation ·

Neuromuscular blockade

\begin{abstract}
Abbreviations
GABA Gamma-aminobutyric acid

SCM Skin conductance measurements
\end{abstract}

\section{Introduction}

A considerable number of papers has been published on a variety of premedication drugs used in term and preterm 
newborns before intubation. The focus of research is usually on the clinical effectiveness of these medications $[15,19]$.

Assessment of pain and stress in newborns is a subject of ongoing investigation since a gold standard does not exist. Several different approaches to quantifying pain in newborns have been developed. These methods rely predominantly on observational techniques and physiological parameters [6, 12].

Skin conductance measurements (SCM) seem to correlate well with observational pain assessment, which has been validated in term and preterm infants $[8,13,20]$. The sympathetic nervous system controls the palmar and plantar sweat glands by secreting acetylcholine in the postganglionic synapses. When activated SCM increase temporarily because of pulsatile sweat excretion, SCM closely follow (with a consistent delay of 1-2 s) the pattern seen during microneurographic recording of sympathetic nerve activation $[2,17]$. An added advantage of this method is that stress assessment is possible in deeply sedated and, reportedly, paralysed (adult) patients [16].

Currently, there are no reports on the effectiveness of premedication drugs on pain and stress reduction in sedated and/or paralysed newborns during painful procedures.

Premedication drugs commonly used prior to intubation of term and preterm newborns include morphine, fentanyl, remifentanil, thiopental, propofol and neuromuscular blockers. The two most frequently used premedication drugs are (1) opioids in combination with a neuromuscular blocker and (2) propofol. There is increasing awareness that morphine might not be very effective in providing analgesia in an emergency setting $[4,19]$. Following the publication of a small RCT, propofol is increasingly used in Dutch NICUs because of its hypnotic activity [11]. However, there are concerns regarding its adverse effects, i.e. its potential for decreasing blood pressure and thus possibly threatening cerebral perfusion [29]. Furthermore, propofol lacks analgesic activity and might not be sufficient as a single premedication drug [25].

In this feasibility study, we investigated the usefulness of $\mathrm{SCM}$ as surrogate marker for stress after premedication with either morphine and vecuronium or propofol during semielective intubation of term and preterm newborns.

\section{Methods}

Newborns requiring semi-elective intubation were prospectively included to receive either morphine+vecuronium or propofol as premedication before naso-tracheal intubation. The reason for using these two different premedication regimens in this setting was that these drugs represent different mechanisms of action for inhibiting stress responses. Randomisation was part of the inclusion procedure for a psychological reason: to prevent group differences regarding medication use by preference of the attending physician. We used a computer-generated randomisation list. An independent research nurse placed group and number in sealed envelopes and numbered these. Upon inclusion, the next numbered envelope was opened.

Inclusion criterion was any newborn developing the need for semi-elective intubation. Exclusion criteria were hypotension or obstructive cardiac outflow tract disorders, suspicion of a metabolic disorder (all propofol-related criteria) or suspicion of a genetic syndrome (attempt at excluding newborns with possible genetic differences in autonomic nervous functioning, i.e. trisomy 21) and the presence of discomfort or pain (presence of a urinary catheter, thorax drain, etc.) or administration of analgetics (opioids, paracetamol) in the preceding $24 \mathrm{~h}$. The research protocol was reviewed and approved by both our institutional medical ethics committees and, since propofol is not registered for use in this age group, also by the Dutch national medical ethics committee.

Informed consent was obtained after the procedure because of the emergent aspect of intubation. In research in emergency settings, retrospective informed consent is fully acceptable in most European countries provided specific criteria are fulfilled $[5,14,22]$.

Patient characteristics, gestational age (GA), postnatal age (PNA), birth weight (BW) and intubation indication, were registered (Table 1).

Medication Morphine and vecuronium were both given as a bolus intravenous injection of $0.1 \mathrm{mg} / \mathrm{kg}$. Propofol $1 \%$ (Lipuro $^{\circledR}$ ) was diluted 1:1 with glucose $5 \%$ in order to diminish pain at the injection site and then slowly administered intravenously in a dose of $2 \mathrm{mg} / \mathrm{kg}$. If necessary, this dose could be repeated once. The dosages of all used medications are according to medication guidelines in Dutch NICUs.

Study protocol SCM (Med-Storm Pain Monitor, Med-storm Innovation AS, Oslo, Norway) were started as soon as the

Table 1 Patient characteristics and procedural parameters (mean $\pm \mathrm{SD}$ )

\begin{tabular}{lll}
\hline Parameter & $\begin{array}{l}\text { Morphine/ } \\
\text { vecuronium }\end{array}$ & Propofol \\
\hline Gestational age (weeks) & $28 \frac{5}{7} \pm 2 \frac{5}{7}$ & $31 \frac{4}{7} \pm 3 \frac{2}{7}$ \\
Birth weight (g) & $1239 \pm 528$ & $1710 \pm 519$ \\
Postnatal age (days) & $1.1 \pm 1.9$ & $0.3 \pm 0.5$ \\
Indication for intubation: IRDS & 8 & 11 \\
Sepsis & 2 & \\
Exhaustion & 1 & \\
Time admin. morphine to T=0 (min) & $6.2 \pm 3.2$ & \\
Time T=0 to start intubation procedure (min) & $0.8 \pm 0.5$ & $0.6 \pm 0.2$ \\
Intubating condition score (max 6) & $5.4 \pm 0.8$ & $4.9 \pm 0.9$ \\
Baseline SCM (peaks/s) & $0.16 \pm 0.02$ & $0.22 \pm 0.03$ \\
\hline
\end{tabular}


decision to intubate had been made. Timelines in the two groups studied were as follows:

Morphine-vecuronium: Baseline SCM recording for at least $60 \mathrm{~s} ; \mathrm{T}=-10 \mathrm{~min}$ : morphine administration; $\mathrm{T}=0$ : vecuronium administration. As soon as the intubating clinician deemed possible, the naso-tracheal intubation procedure was started. Intubating conditions were scored using a modified intubating score [10] (Table 2).

Propofol: Baseline SCM recording for at least $60 \mathrm{~s} ; \mathrm{T}=0$ : propofol administration. As soon as the intubating conditions were acceptable, the naso-tracheal intubation procedure was started. Intubations were performed by nurse practioners, residents or neonatologists, reflecting the setting of a major teaching hospital.

Skin conductance measurements (SCM) The following specific events were recorded: the moment the medication was administered, any painful or stressful stimuli (removal of adhesive plaster, suction, non-invasive ventilation, insertion of tube and laryngoscope, and the moment intubation was completed successfully. Measurements were recorded continuously up to $30 \mathrm{~min}$ after completion of the intubation procedure. The parameter used for assessing SCM was the number of peaks/s, which is the best validated skin conductance parameter for pain score in newborns. This parameter represents the number of peaks within a time window and corresponds to the number of bursts per second in a skin sympathetic nerve $[2$, 17]. When correlated with Prechtl's general movement observations, a cut-off point of 0.21 peaks $/ \mathrm{s}$ reflects the threshold at which stress may become unacceptable [24]. We recorded the number of peaks/s in consecutive time windows of $15 \mathrm{~s}$. A group size of ten patients in each group was deemed sufficient for assessment of feasibility of SCM during intubation.

Physiological parameters Heart rate, pulse oximetry and arterial blood pressure (in case an arterial indwelling catheter was present) were continuously recorded on a central monitoring system up to $30 \mathrm{~min}$ after completion of the intubation procedure. When no indwelling arterial catheter was present, blood pressure was measured non-invasively every $5 \mathrm{~min}$.
Data analysis All data were analysed using the software program GraphPad Prism 5.01 (GraphPad Software Inc., San Diego, CA, USA). The differences in the number of peaks/s at the various time points of the procedure during each procedure were analysed statistically, using the non-parametric oneway ANOVA test; Dunn's multiple comparison test was performed in order to find statistical differences between each of the analysed time points.

\section{Results}

Originally, 20 newborns were to be included and randomised. However, due to technical problems with data storage on the SCM apparatus, the randomisation period was extended after conferring with our institutional medical ethics committee. In total, 28 newborns were included and randomised; in two cases, informed consent was denied. Of the 26 remaining newborns, 13 received morphine and vecuronium and 13 received propofol as premedication. In both groups, two recordings were lost due to technical difficulties and these patients were excluded. A total of 22 newborns (11 in each group) were analysed. Patient characteristics and procedural parameters are presented in Table 1. In each group, one patient exhibited a very high baseline and a significantly higher number of peaks/s both at rest and during painful stimuli resulting in higher standard deviations of the means without significant effect on the means. Interindividual variation was substantial as reflected by the coefficient of variation for the baseline values: $61 \pm 49 \%$ (range $0-141 \%$ ) and $50 \pm 54 \%$ (range 0 $200 \%$ ).Also intraindividual variation was present as reflected in Fig. 1: not all increase in peaks in SCM can be explained by the applied stimuli. Morphine was administered $6.2 \pm 2.7 \mathrm{~min}$ (mean \pm SD; range 1.4-10.3 $\mathrm{min}$ ) before vecuronium instead of at least $10 \mathrm{~min}$ before vecuronium as described in our protocol (in nine patients, vecuronium was administered earlier than 10 min after morphine administration). Clinical deterioration and the emergent nature of the procedure were given as the reasons for this breach of protocol. Administration of morphine before administration of vecuronium did not have any effect on SCM when a stressful stimulus (not related to intubation; for instance, removal of adhesive tape) was applied
Table 2 Intubating condition score (ICS)

\begin{tabular}{llll}
\hline & \multicolumn{2}{l}{ Clinically acceptable } & Not clinically acceptable \\
\cline { 2 - 3 } & Excellent (2) & Good (1) & Bad (0) \\
\hline Reaction to introduction tube & No & Slight & Vigorous/sustained \\
Laryngoscopy & Easy & Fair & Difficult \\
Position of vocal cords & Abducted & Intermediate/moving & Closed \\
\hline
\end{tabular}

Adapted from Fuchs-Buder et al. (2007). Acceptable: ICS 3 or higher 


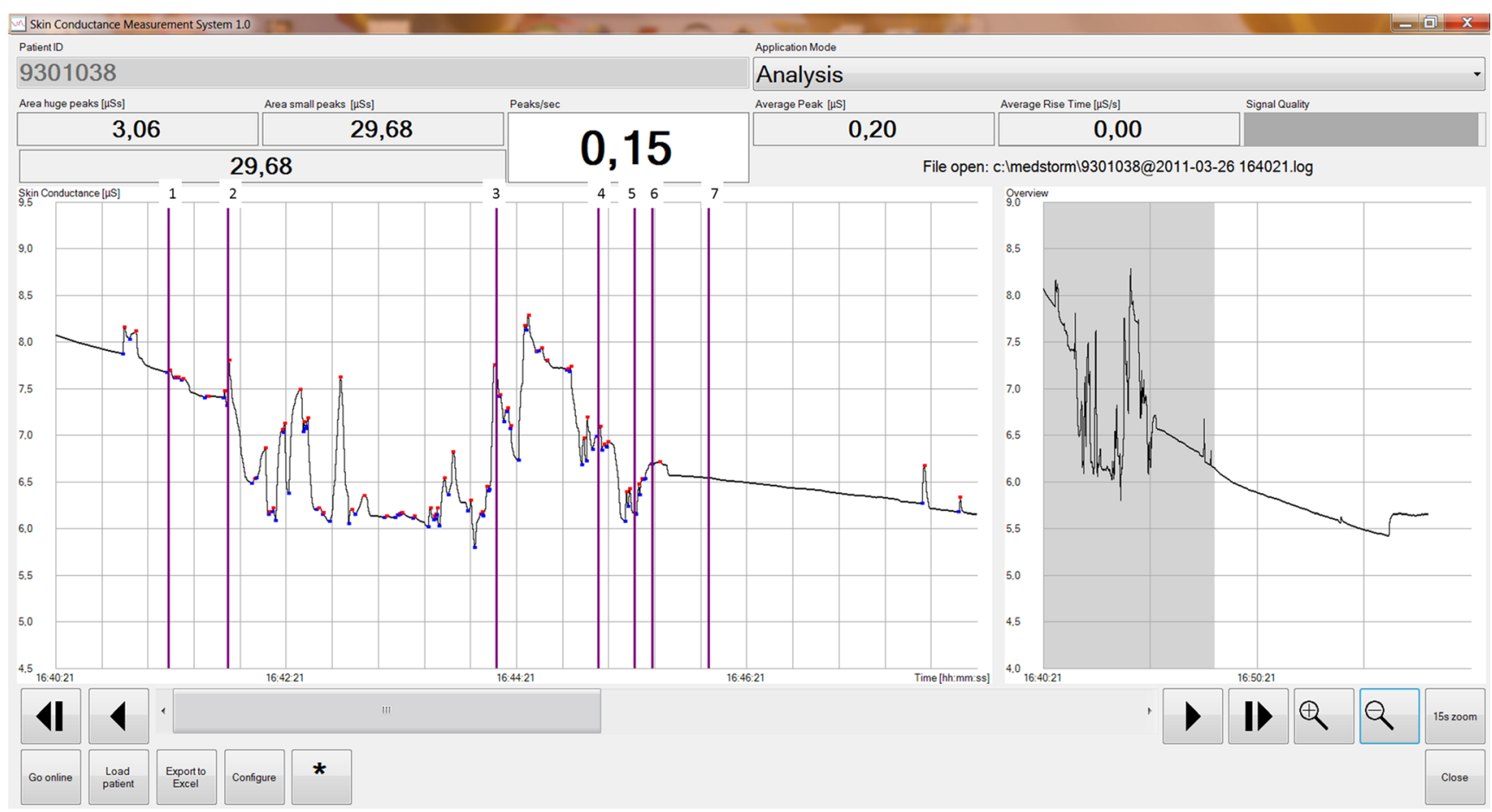

Fig. 1 Graphical presentation of skin conduction (mean peaks/s \pm SD) in relation to events in all patients in the morphine/vecuronium group. Each colour represents the number of patients (between brackets) intubated at

(median 0.18 peaks/s; range $0.00-0.80$ peaks/s). Within $1.6 \mathrm{~min}$ (range $0.8-3 \mathrm{~min}$ ) after administration of vecuronium, SCM disappeared in 10 of 11 newborns (median 0.00 peaks/s; range $0.00-0.27$ peaks $/ \mathrm{s}$ ). This precluded further analysis of the effect of morphine on SCM during the intubation procedure (Fig. 2). Propofol was effective in reducing SCM in 10 of 11 newborns at the first attempt (median 0.04 peaks/s; range $0.00-0.33$ peaks $/ \mathrm{s}$ ). Further attempts were associated with increasing SCM, mostly above a threshold of 0.21 peaks $/ \mathrm{s}$ which were not influenced by a second dose of propofol (Fig. 3). Intubating condition scores remained clinically acceptable throughout the procedure, but for one patient in the propofol group (the only patient intubated after the fourth attempt). Analysis of blood pressure, heart rate and oxygen saturation revealed no significant differences from baseline measurements (data not shown). One patient receiving propofol required a fluid bolus because of arterial hypotension.

\section{Discussion}

The two main findings in this study were as follows:

1. Significant variation in SCM both between individual as within individual patients is present in comparable conditions. the first, second or third attempt, respectively. The high SD values reflect substantial interindividual variation

2. Neuromuscular blockade with vecuronium reduces SCM to zero within approximately $3 \mathrm{~min}$.

We observed temporal relations between possible stressful stimuli and SCM in 20 out of 22 analysed patients. This temporal relationship has been reported by other authors $[8,16$, 20]. However, in each group, one patient displayed a significantly higher baseline activity that was only minimally reduced by premedication regimens or increased by stimuli. Interestingly, these patients reacted clinically similar to the other patients. The expected interindividual variability in sympathetic tone is apparently reflected in a significant variance in skin conductivity. Intraindividual variation was also present as depicted in Fig. 1: Peaks in SCM appear not only after (potential) stressful stimuli but also spontaneously. Both interindividual and intraindividual variation of SCM might make it difficult to draw conclusions from the measurements in clinical situations.

Morphine is an analgesic opiate with mild sedative effect when given continuously [1]. In the acute setting, the effectiveness is questionable, most likely due to its relatively slow onset of action. This might explain that responsiveness to stressful stimuli before intubation still resulted in increased SCM. After vecuronium administration, the SCM all but disappeared within $3 \mathrm{~min}$, precluding further analysis of the effects of morphine on SCM. The unexpected decline in SCM after administration of vecuronium is very difficult to explain. Although morphine might play a role in the disappearance of 


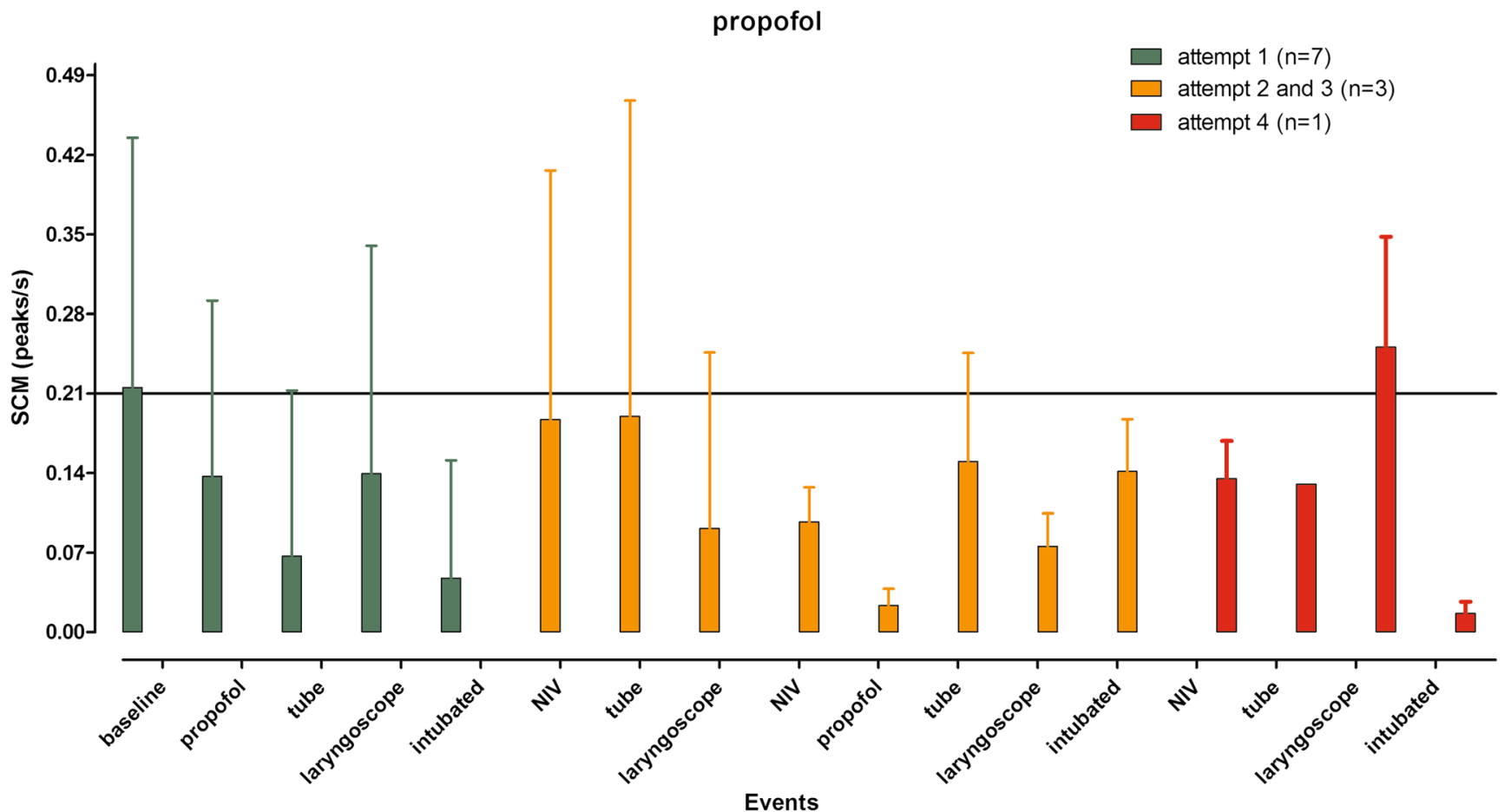

Fig. 2 Graphical presentation of skin conduction (mean peaks $/ \mathrm{s} \pm \mathrm{SD}$ ) in relation to events in all patients in the propofol group. Each colour represents the number of patients (between brackets) intubated at the

first, third (no second attempt was successful) or four attempt, respectively. The high SD values reflect substantial interindividual variation

SCM after administration of vecuronium, this is not likely, both because of its slow onset of action and the universal

disappearance of SCM regardless of the time interval between morphine and vecuronium administration. In adult patients,

Morphine / Vecuronium

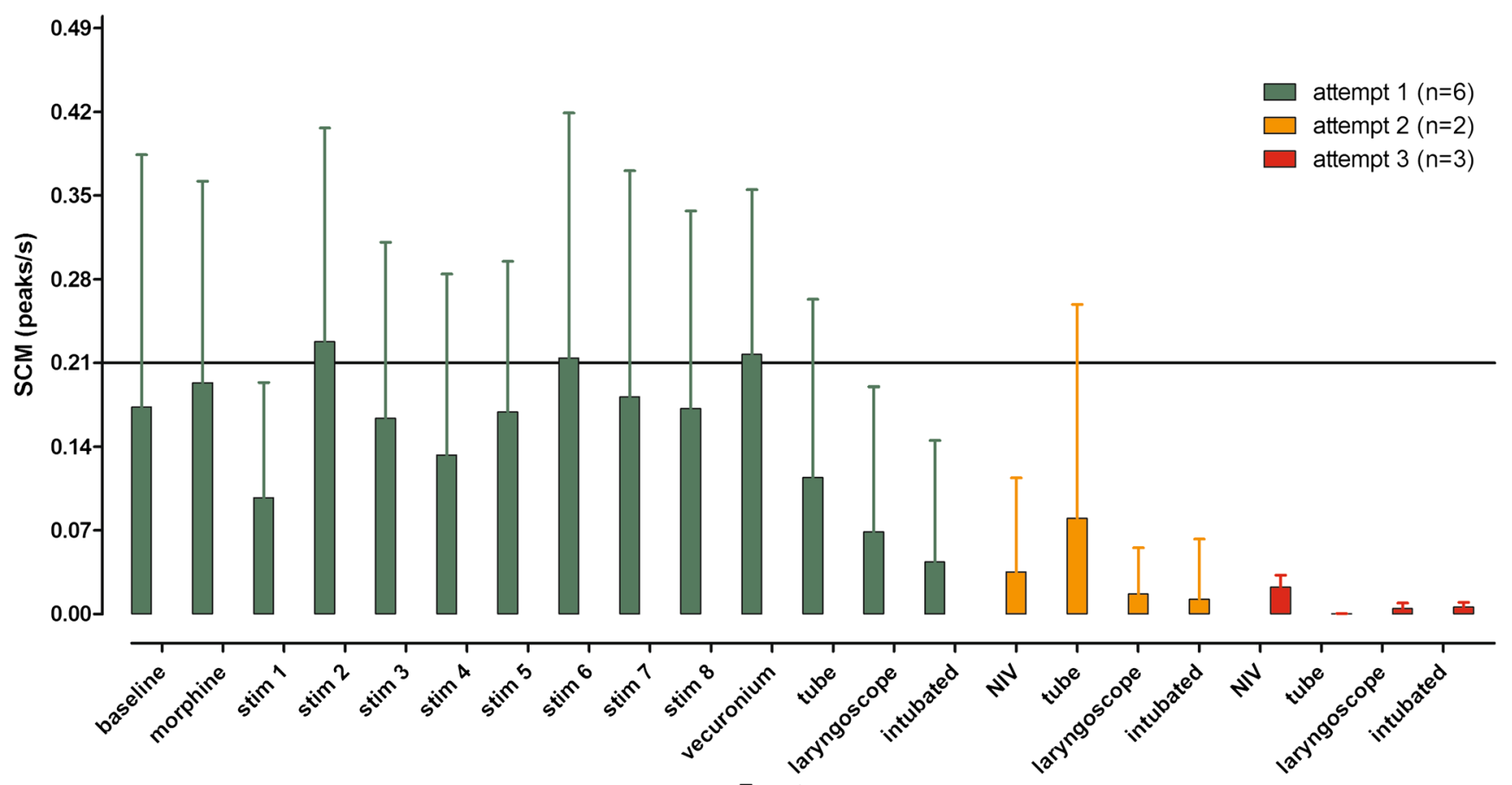

Events

Fig. 3 Representative recording of a patient in the MV group. The blue and red dots represent peaks in SCM on a baseline of conductance $(\mu \mathrm{S})$. Purple lines are annotations of events: 1, morphine administration; 2, repositioning of the infant; 3 , removal of adhesive tape; 4 , vecuronium administration; 5, nasal insertion of the tube; 6 , introduction of laryngoscope; 7 , intubated 
no disappearance of SCM is observed during intubation with sedatives and rocuronium [16]. Therefore, immaturity of presynaptic or postsynaptic receptors involved in palmar sudomotor activation is most likely the reason for the observed effect. Unfortunately, although maturation has been described in the literature for various receptors, including the postsynaptic nicotinic Ach- $\mathrm{R}$, no specific information is available on maturation of the sudomotor response [9]. Muscarinic (M3) receptors are involved in the activation of the sudomotor response and pathways for noradrenergic involvement have been documented [27, 28]. In 1994, Norel et al. showed that vecuronium could inhibit M3 receptors in human airways [18]. However, the absence of a measurable effect of neuromuscular blockade on SCM in adult patients seems to indicate that direct M3 inhibition at the sudomotor junction cannot be held accountable for the observed effect in premature babies. To complicate matters, SCM involves not just the sudomotor response but also the sympathetic vasomotor response [7]. In much earlier publications, galvanic skin responses could still be recorded in patients with congenital absence of sweat glands and even post mortem, obscuring the picture of the physiology behind SCM even further [21, 23, 26]. In summary, there is a robust relation between SCM and sympathetic nerve activity but which of the several mechanisms involved in SCM is affected by administration of vecuronium in our premature babies remains unclear.

Propofol inhibits the sympathetic nervous system centrally and causes deep hypnosis (primarily, but not exclusively, via the gamma-aminobutyric acid $\left(\mathrm{GABA}_{\mathrm{A}}\right)$ system) but has no known analgesic effect. In newborns, the neuronal chloride gradient which is critical to $\mathrm{GABA}_{\mathrm{A}}$ function is still immature: stimulation of $\mathrm{GABA}_{\mathrm{A}}$ receptors then causes excitation instead of the inhibitory response seen in the mature condition. Maturation of $\mathrm{GABA}_{\mathrm{A}}$ receptors (reversal of the chloride gradient) occurs in a caudo-rostral direction. It is known that in neonatal convulsions, treatment with benzodiazepines usually leads to cessation of clinically apparent convulsions while an EEG can still show epileptic activity [3]. Analogous to this observation, dissociation between peripheral and central $\mathrm{GABA}_{\mathrm{A}}$ responsiveness might well explain our observations: initially adequate sedation with good muscle relaxation. However, as the procedure continued muscle relaxation persisted (more mature $\mathrm{GABA}_{\mathrm{A}}$ ), accounting for clinically acceptable intubation conditions. At the same time, central responsiveness decreased or reverted to a more excitatory state which might explain increasing SCM.

This study shows several weaknesses. This study was clearly underpowered for a thorough analysis of the unexpected findings. Specifically, the relationship between maturation (and/or postnatal age) and the unexpected almost complete block of SCM by vecuronium could not be analysed in depth. Although very interesting the unexpected findings did not warrant an increase of the size of the groups as these findings would not answer our original question. The lack of conforming to study protocol regarding the time between the administration of morphine and vecuronium was unfortunate but in accordance with the clinical situation of impending respiratory failure.

\section{Conclusions}

The observed interindividual and intraindividual variation in SCM in our newborns limits the usefulness of SCM as stress monitor during intubation. The use of neuromuscular blockers for premedication precludes monitoring of SCM completely in newborns.

\section{Compliance with ethical standards}

Funding No funds were received for this study.

Author's contribution All listed authors are responsible for the reported research. Dr R. van der Lee wrote the first draft of the manuscript. Dr R. van der Lee, Dr. R.A. van Lingen and Mrs L.J.M. Groot-Jebbink were responsible for analysis and interpretation of the data. All authors participated in the concept and design and in drafting or revising the manuscript; all authors approved the manuscript as submitted.

Conflict of interest Author R. van der Lee declares that he has no conflict of interest. Author Liesbeth JM Groot-Jebbink declares that she has no conflict of interest. Author Thea HM van Herpen declares that she has no conflict of interest. Author Esther J d'Haens declares that she has no conflict of interest. Author Josette Bierhuizen declares that she has no conflict of interest. Author Richard A van Lingen declares that he has no conflict of interest.

Ethical approval All procedures performed in studies involving human participants were in accordance with the ethical standards of the institutional and/or national research committee and with the 1964 Helsinki declaration and its later amendments or comparable ethical standards.

Informed consent Informed consent was obtained from all individual participants included in the study.

Open Access This article is distributed under the terms of the Creative Commons Attribution 4.0 International License (http:// creativecommons.org/licenses/by/4.0/), which permits unrestricted use, distribution, and reproduction in any medium, provided you give appropriate credit to the original author(s) and the source, provide a link to the Creative Commons license, and indicate if changes were made.

\section{References}

1. Bellu R, de Waal K, Zanini R (2010) Opioids for neonates receiving mechanical ventilation: a systematic review and meta-analysis. Arch Dis Child Fetal Neonatal Ed 95(4):F241-F251

2. Bini G, Hagbarth K-E, Hynninen P, Wallin BG (1980) Thermoregulatory and rhythm-generating mechanisms governing the sudomotor and vasoconstrictor outflow in human cutaneous nerves. J Physiol 306:537-552 
3. Briggs SW, Galanopoulou AS (2011) Altered GABA signaling in early life epilepsies. Neural Plast 2011: Article ID 52760516 pages doi: $10.1155 / 2011 / 527605$

4. Carbajal R, Lenclen R, Jugie M, Paupe A, Barton BA, Anand KJS (2006) Morphine does not provide adequate analgesia for acute procedural pain among preterm newborns. Pediatrics 115:1494-1500

5. Dalla-Vorgia P, Mason S, Megone C, Allmark P, Bratlid D, Gill AB, Morrogh P, Plomer A, Reiter-Theil S (2001) Overview of European legislation on informed consent for neonatal research. Arch Dis Child Fetal Neonatal Ed 84:F70-F73

6. Donadio V, Lenzi P, Montagna P, Falzone F, Baruzzi A, Liguori R (2005) Habituation of sympathetic sudomotor and vasomotor skin responses: neural and non-neural components in healthy subjects. Clin Neurophysiol 116:2542-2549

7. Eriksson M, Storm H, Fremming A, Scholling J (2008) Skin conductance compared to a combined behavioural and physiological pain measure in newborn infants. Acta Paediatr 97:27-30

8. Fagerlund MJ, Eriksson LI (2009) Current concepts in neuromuscular transmission. Br J Anaesth 103:108-114

9. Fuchs-Buder T, Claudius C, Skovgaard LT, Eriksson LI, Mirakhur RK, Viby-Mogensen J (2007) Good clinical research practice in pharmacodynamic studies of neuromuscular blocking agents II: the Stockholm revision. Acta Anaesthesiol Scand 51:789-808

10. Ghanta S, Abdel-Latif ME, Lui K, Ravindranathan H, Awad J, Oei J (2007) Propofol compared with the morphine, atropine and suxamethonium regimen as induction agents for neonatal endotracheal intubation: a randomized controlled trial. Pediatrics 119: e1248-e1255

11. Gibbins S, Stevens B, McGrath PJ, Yamada J, Beyene J, Breau L, Camfield C, Finley A, Franck L, Johnston C, Howlett A, McKeever $\mathrm{P}$, O'Brien K, Ohlsson A (2008) Comparison of pain responses in infants of different gestational ages. Neonatology 93:10-18

12. Hellerud BC, Storm H (2002) Skin conductance and behaviour during sensory stimulation of preterm and term infants. Early Hum Dev 70:35-46

13. Jansen TC, Kompanje EJO, Bakker J (2009) Deferred proxy consent in emergency critical care research: ethically valid and practically feasible. Crit Care Med 37(Suppl):S65-S68

14. Kumar P, Denson SE, Mancuso TJ, Committee on Fetus and Newborn, Section on Anesthesiology and Pain Medicine (2010) Premedication for nonemergency endotracheal intubation in the newborn. Pediatrics 125:608-615

15. Ledowski T, Pascoe E, Ang B, Schmarbeck T, Clarke MW, Fuller C, Kapoor V (2010) Monitoring of intra-operative nociception: skin conductance and surgical stress index versus stress hormone plasma levels. Anaesthesia 65:1001-1006
16. Macefield VG, Wallin BG (1996) The discharge behaviour of single sympathetic outflow in normotensive human sweat glands. J Auton Nerv Syst 14:277-286

17. Norel X, Labat C, DeSantis D, Gorenne I, Dulmet E, Rossi F, Brink C (1994) Cholinesterase inhibition by vecuronium and pancuronium in human airways. Life Sci 55(14):261-266

18. Norman E, Wikstrom S, Hellstrom-Westas L, Turpeinen U, Hamalainen E, Fellman V (2011) Rapid sequence induction is superior to morphine for intubation of preterm infants: a randomized controlled trial. J Pediatr 159:893-899

19. Pereira-da-Silva L, Virella D, Monteiro I, Gomes S, Rodrigues P, Serelha M, Storm H (2012) Skin conductance indices discriminate nociceptive responses to acute stimuli from different heel prick procedures in infants. J Matern Fetal Neonatal Med 25:796-801

20. Richter CP (1927) A study of electrical skin resistance and the psychogalvanic reflex in a case of unilateral sweating. Brain 50: 216-235

21. Schellings R, Kessels AG, Ter Riet G, Sturmans F, Widdershoven GA, Knottnerus JA (2009) Indications and requirements for the use of prerandomization. J Clin Epidemiol 62:393-399

22. Shaver BA, Brusilow SW, Cooke RE (1962) Origin of the galvanic skin response. Proc Soc Exp Biol NY 110:559-564

23. Storm H. Pain assessment in newborns (2012) In: Chen W, Oetomo SB, \& Feijs L Neonatal monitoring technologies: design for integrated solutions. Hershey, IGI Global. doi: 10.4018/978-1-46660975-4 278-302

24. TerRiet MF, Jacobs JS, Lewis MC, DeSouza GJA (2000) Propofol and analgesia (letter). Anesth Analg 90:1455

25. van Dijk M, Roofthooft DWE, Anand KJS, Guldemond F, de Graaf J, Simons S, de Jager Y, van Goudoever JB, Tibboel D (2009) Taking up the challenge of measuring prolonged pain in (premature) neonates. Clin J Pain 25:607-616

26. Vetrugno R, Liguori R, Cortelli P, Montagna P (2003) Sympathetic skin response - basic mechanisms and clinical applications. Clin Auton Res 13:256-270

27. Vilches JJ, Navarro X, Verdu E (1995) Functional sudomotor responses to cholinergic agonists and antagonists in the mouse. $\mathrm{J}$ Auton Nerv Syst 55:105-111

28. Weihe E, Schutz N, Hartschuh W, Anlauf M, Schafer MK, Eiden LE (2005) Coexpression of cholinergic and noradrenergic phenotypes in human and nonhuman autonomic nervous system. J Comp Neurol 492:370-379

29. Welzing L, Kribs A, Eifinger F, Huenseler C, Oberthuer A, Roth B (2010) Propofol as an induction agent for endotracheal intubation can cause significant arterial hypotension in preterm newborns. Paediatr Anesth 20:605-611 